\title{
Spectrum of the WHO Classification De Novo Myelodysplastic Syndrome: Experience from Southern Pakistan
}

\author{
Sadia Sultan ${ }^{1 *}$, Syed Mohammed Irfan', Syeda Narisa Jawed ${ }^{2}$
}

\begin{abstract}
Background: Myelodysplastic syndrome (MDS) is a clonal disorder of hemopoeitic stem cells, characterized by infective hematopoiesis, peripheral cytopenias along with hypercellularity of marrow and marked dysplastic features. Our aim was to study the spectrum of the WHO classification in adult Pakistani patients with MDS at disease presentation. Materials and Methods: This retrospective descriptive study was conducted at Liaquat National Hospital and Medical College, extending from January 2010 to December 2014. Patient data were retrieved from the maintained archives. Results: Overall, 45 patients were diagnosed at our institution with de novo MDS during the study period. There were 28 males and 17 females. Age ranged between 18 and 95 years with a mean of 57.6 \pm 17.4 years. The male to female ratio was 1.7:1. According to the WHO classification, 53.3\% had refractory cytopenia with multilineage dysplasia, $22.2 \%$ had refractory cytopenia with unilineage dysplasia, 4.4\% each had refractory anemia with excess of blasts-1 and II and 15.5\% had MDS unclassified. The main presenting complaints were generalized fatigue $(60 \%)$, fever $(33.3 \%)$, dyspnea $(15.5 \%)$, bleeding $(13.3 \%)$ and weight loss $(11.1 \%)$. Physical examination revealed pallor in $37.7 \%$, followed by petechial and purpuric rashes in $20 \%$ of patients. Hemoglobin was $<10 \mathrm{~g} / \mathrm{dl}$ in $41(91.1 \%)$. Pancytopenia and bicytopenia were noted in $18(40 \%)$ and $14(31.1 \%)$ respectively. Conclusions: MDS in our patients presents at a relatively young age. Refractory cytopenia with multilineage dysplasia was the dominant disease variant in our setting.
\end{abstract}

Keywords: Myelodysplastic syndrome - WHO classification - adults - Pakistan

Asian Pac J Cancer Prev, 17 (3), 1049-1052

\section{Introduction}

Myelodysplastic syndromes (MDS) are a heterogeneous group of clonal stem cell disorders characterized by active but ineffective hematopoiesis, cytopenias with hypercellular bone marrow and marked dysplasia (Newman et al., 2012; Akinci et al., 2014). MDS has been recognized for more than 50 years and has been identified as a preleukemia state with an increased risk of AML transformation. In general $40 \%$ of patients will transform to AML during the disease course (Chevassut et al., 2011; Gao et al., 2015).

The available literature revealed an incidence of MDS as $3-5 / 100,000$ populations annually that increases to 20 to $50 / 100000$ persons yearly above 60 years of age (Bernasconi et al., 2005; Malcovati et al., 2013; Rashid et al., 2014). It generally occurs in older adults with the median age of $\sim 70$ but may also affect children (Rodrigues et al., 2007; Neukirchen et al., 2011).

The presence of a malignant clone is reflected by marrow dysplasia and an impaired functional activity which might affect all the three hematopoietic cell lineages. In the last two decades there have been escalations in our understanding of MDS after recognization of molecular lesions.

The diagnosis of MDS has always been a controversial and challenging. Recent categorization of MDS as per WHO classification include refractory cytopenia with unilinage dysplasia, refractory anemia with ring sideroblast, refractory cytopenia with multilineage dysplasia, refractory anemia with excess blast-1 \& 2, myelodysplastic syndrome unclassified and MDS associated with isolated del (5q) (Brunning et al., 2008).

Because of difficulties in diagnosis and data recording nationally, the epidemiological features of MDS are still poorly defined. To date, there is no tumor registry maintained in Pakistan to keep a record of regional occurrence of MDS. The present study is designed to determine the current spectrum of MDS subtypes in Pakistani patients with myelodysplasic syndrome according to WHO classification.

\section{Materials and Methods}

This retrospective analysis was carried out at the Department of Haematology, Liaquat National Hospital Karachi, extended from January 2010 to December 2014.

Patients data were retrieved from the maintained 
Table 1. WHO Classification of Adults Patients with MDS in Pakistani Studies

\begin{tabular}{|c|c|c|c|c|c|c|c|}
\hline Author/year & RCUD & RCMD & RAEB-1 & RAEB-II & RARS & RCMD-RS & Unclassified \\
\hline $\begin{array}{l}\text { Ehsan et al } \\
2010 \\
\text { Lahore }\end{array}$ & 26.1 & 52.2 & 6.5 & 6.5 & 0 & 2.2 & 6.5 \\
\hline $\begin{array}{l}\text { Rashid et al } \\
2014 \\
\text { Karachi }\end{array}$ & 8.5 & 52 & 11.3 & 23.9 & 2.8 & 1.4 & 0 \\
\hline $\begin{array}{l}\text { Present study } \\
2015 \\
\text { Karachi }\end{array}$ & 22.2 & 53.3 & 4.4 & 4.4 & 0 & 0 & 15.5 \\
\hline
\end{tabular}

archives. We studied a cohort of 45 patients with newly diagnosed adults MDS. Complete clinical details and laboratory data were available on all patients.

The diagnosis of MDS was ascertained according to the standard WHO criteria and was based on peripheral cytopenias, bone marrow dysplasia and percentage of blast cell counts (Brunning et al., 2008).

Complete blood counts were analyzed by automated hematology analyzer Cell Dyne Ruby (Abott, Diagnostics). All peripheral blood smears were reviewed by expert hematopathologists. Bone marrow samples were taken from posterior iliac crest through Jamshidi needle and were stained by Leishman's stain. Perl's stain was carried out on each bone marrow smears by commercially provided kits from Merck Diagnostic according to manufacturer's instructions.

The ethical endorsement of the study was granted by research and ethical committee LNH taken prior to the study.

\section{Data analysis}

Statistical Package for the Social Sciences version 22.0 (SPSS Inc, Chicago, IL, USA) was used for statistical analysis. Descriptive variables was presented as mean (SD). Data was presented as frequencies and percentages.

\section{Results}

\section{Demographical profile}

Of total 45 patients, 28 were males (62.2\%) and 17 were females $(37.7 \%)$. The male to female ratio was $1.7: 1$. The mean age of patients at presentation was 57.6 \pm 17.4 (range 18-95) years with the median age of 64 years.

\section{Clinical and laboratory findings}

The main presenting complaints were generalized fatigability (60\%); fever (33.3\%); dyspnea (15.5\%); bleeding $(13.3 \%)$ and weight loss $(11.1 \%)$. Examination was unremarkable in majority $(42.2 \%)$ of patients. Physical examination revealed pallor in $37.7 \%$ of patients, followed by petechial and purpuric rashes in $20 \%$ of patients.

The mean hemoglobin was $7.7 \pm 2.4 \mathrm{~g} / \mathrm{dl}$. The mean total leukocyte count of $5.7 \pm 5.8 \times 109 / 1$; mean absolute neutrophilic count of $3.0 \pm 5.4 \times 10^{9} / 1$ and the mean platelets count were $82.7 \pm 104.6 \times 10^{\%} / 1$. The commonest laboratory findings was anemia; Hemoglobin $<10 \mathrm{~g} / \mathrm{dl}$ in $41(91.1 \%)$ patients. Out of which 27 (60\%) patients had normocytic anemia. Pancytopenia and bicytopenia was noted in 18
(40\%) and $14(31.1 \%)$ patients respectively.

\section{WHO classification of MDS}

According to the WHO classification, 24 (53.3\%) patients had refractory cytopenia with multilineage dysplasia (RCMD); 10 (22.2\%) patients had refractory cytopenia with unilineage dysplasia (RUCD); 2 (4.4\%) each of refractory anemia with excess of blasts (RAEB) I and II and 7 (15.5\%) patients had MDS unclassified.

In RCUD variant, 8 (17.7\%) patients had refractory anemia (RA) while $2(4.4 \%)$ patients had refractory thrombocytopenia (RT). None of our patient had refractory neutropenia $(\mathrm{RN})$.

\section{Discussion}

Myelodysplastic syndrome is a heterogeneous disease with the significant biological and clinical differences amongst the various disease variants (Shah et al., 2009). This hematological disorder is not uncommon but mostly under reported from our part of the world. As limited studies are available from Pakistan on MDS (Irfan et al., 1998; Ehsan et al., 2010; Rashid et al., 2014). Herein we have described a series of 45 adults MDS patients followed at our institution during the five year period. MDS has been considered as a disease of the elder but in Pakistani population it is identified in younger age as compared with west (de Hollanda et al., 2011; Fenaux et al., 2014).

Internationally, there are two standard classification systems that are widely used for the diagnosis of primary Myelodysplasia (MDS). According to the French American British classification (FAB) system, MDS is diagnosed in patients who have $<30 \%$ blasts in bone marrow and peripheral blood along with evidence of dysplasia. However, significant heterogeneity has been noted within FAB subgroups. Recent classification by the World Health Organization (WHO) reduces the percentage of blasts from $30 \%$ to $20 \%$, taking into consideration, the pertinent fact that patients with $20 \%$ to $30 \%$ blasts do have AML (Brunning et al., 2008).

The WHO classification of MDS has been comprehensively studied at international levels but not at national level. Earlier only two regional studies were available from Pakistan (Ehsan et al., 2010; Rashid et al., 2014). We determined MDS-RCMD (53.3\%) as the commonest type, followed by RCUD (22.2\%). Previously, published studies from Pakistan also revealed RCMD as a major subtype (Ehsan et al., 2010; Rashid et al., 2014). Table-1 depicted the WHO distribution in prior studies on 
Pakistani patients with adults MDS.

When compared these findings with the two Indian studies, the predominant subtype was RA (37.5\% and 55.7\%) (Kar et al., 2009; Chaubey et al., 2011). However, Chatterjee et al from India revealed RCMD as a predominant subtype accountable in $69.6 \%$ of their study subjects (Chatterjee et al., 2004). Similarly, studies from China reported the predominance of RCMD catogory in Chinese MDS patients (Wang et al., 2008; Yan et al., 2015).

Akiba et al from Japan showed RCUD-RA (33\%) as a common subtype in Japanese patients with MDS (Akiba et al., 2001). Present study also confirms RA (17.7\%) as the second frequent occurring subtype in Pakistani patients. It can be easily appreciated that RCMD and RA together constituted the major disease in our study, which is in concurrence with other studies under consideration. It is note worthy that in studies from South East Asia (India, China and Pakistan) the number of RCMD patients comprised the major bulk of the disease, suggesting a similar disease epidemiology in this region. The specific MDS subtype (RCMD) may be due to the some underlying genetic factors which may be responsible for a particular subtype in our population.

A number of biological and clinical factors affect the outcome in patients with MDS. The differences in prognosis have been observed among the different WHO subtypes. Generally cases of RAEB I \& II have a worse prognosis than those of RUCD and RA.

Luckily, RAEB I and II were relatively uncommon in the present study, similar findings were reported in other studies as well. RAEB is an oligoblastic leukemia, carrying a higher probability of evolving into acute leukemia with median survival of less than 24 months. The more advanced the disease at the time of treatment, the worse will be the outcome.

A limitation of the study includes lack of karyotyping testing due to which del 5q- cases were not identified. Secondly, lack of follow up data was a restricting factor on elaborating the disease outcome and clinical course. It is highly suggested that larger prospective studies should be carried out with cytogenetic evaluation and follow up information to understand the spectrum of MDS in Pakistani setup.

In conclusion, WHO classification system served to discriminate several disease subgroups with well defined clinical course. The studied cohort of Pakistani MDS had a relatively younger age with normocytic anemia as a most common clinical finding. Virtually half of patients had refractory cytopenia with multilineage dysplasia as a major variant in our patients. Future studies should be pursueded on a larger patients series to explicate further disease spectrum and definitely should incorporate prognostic karyotyping and molecular testing to determine the IPSS scoring in Pakistani population.

\section{References}

Akiba M, Matsuda A, Misumi M, ET AL (2001). Clinical significance of WHO classification and MDS 2000 classification in myelodysplastic syndromes. Rinshu Ketsuk,
42, 162-9.

Akinci S, Silay K, Ulas A, et al (2014). Neutrophil to lymphocyte ratio--not an independent prognostic factor in patients with the myelodysplastic syndrome. Asian Pac J Cancer Prev, 15, 10883-5.

Bernasconi P, Klersy C, Boni M, et al (2005). Incidence and prognostic significance of karyotype abnormalities in de novo primary myelodysplastic syndromes: a study on 331 patients from a single institution. Leukemia, 19, 1424-31.

Brunning RD, Orazi A, Germing U, Le Beau MM (2008). Myelodysplastic syndromes/neoplasms, overview. In: Swerdlow HS, Campo E, Haris LN, et al. WHO Classification of Tumours of haemopoietic and lymphoid tissues. Lyon: International agency for research on cancer, 89.

Chevassut TJ, Mufti GJ (2011). The myelodysplastic syndromes. In:Hoffbrand AV, Catovsky D, Tuddenham EG, Green AR. Post Graduate Hematology, $6^{\text {th }}$ edition, 503.

Chaubey R, Sazawal S, Dada R, Mahapatra M, Saxena R (2011). Cytogenetic profile of Indian patients with de novo myelodysplastic syndromes. Indian J Med Res, 134, 452-7.

Chatterjee T, Dixit A, Mohapatra M, et al (2004). Clinical, haematological and histomorphological profile of adult myelodysplastic syndrome: study of 96 cases in a single institute. Eur J Haematol, 73, 93-7.

de Hollanda A, Beucher A, Henrion D, et al (2011). Systemic and immune manifestations in myelodysplasia: a multicenter retrospective study. Arthritis Care Res, 63, 1188-94.

Ehsan A, Aziz M (2010). Clinico-haematological characteristics in Pakistani patients of primary myelodysplastic syndrome according to World Health Organization classification.J Coll Physicians Surg Pak, 20, 232-6.

Fenaux P, Haase D, Sanz GF, et al(2014). ESMO Guidelines Working Group. Myelodysplastic syndromes: ESMO Clinical Practice Guidelines for diagnosis, treatment and follow-up. Ann Oncol, 25, 57-69.

Gao S, Li Z, Fu JH, et al (2015). Decitabine in the treatment of acute myeloid leukemia and myelodysplastic syndromes, which combined with complex karyotype respectively. Asian Pac J Cancer Prev, 16, 6627-32.

Irfan M, Kakepoto GN, Khursheed M (1998).Primary myelodysplastic syndrome: clinical spectrum of 53 cases. $J$ Pak Med Assoc, 48, 69-73.

Kar R, Rao S, Saxena R (2009). Myelodysplastic syndromes: classification and prognostic scoring systems and their applicability in Indian scenario-experience from a tertiary care center. Hematol, 14, 145-9.

Malcovati L, Hellstrom-Lindberg E, Bowen D, et al (2013). Diagnosis and treatment of primary myelodysplastic syndromes in adults: recommendations from the European Leukemia Net. Blood, 122, 2943-64.

Newman K, Maness-Harris L, El-Hemaidi I, Akhtari M (2012). Revisiting use of growth factors in myelodysplastic syndromes. Asian Pac J Cancer Prev, 13, 1081-91.

Neukirchen J, Schoonen WM, Strupp C, et al (2011). Incidence and prevalence of myelodysplastic syndromes: data from the Dusseldorf MDS-registry. Leuk Res, 35, 1591-6.

Rodrigues EF, de Souza DC, Camargo A, et al (2007). Cytogenetic biclonality in a child with hypocellular primary myelodysplastic syndrome. Cancer Genet Cytogenet, 178, 70-2.

Rashid A, Khurshid M, Shaikh U, Adil S (2014). Chromosomal abnormalities in primary myelodysplastic syndrome. J Coll Physicians Surg Pak, 24, 632-5.

Shah NM, Prajapati SG, Adesara RP, Patel AP (2009). An analysis of 30 cases of myelodysplastic syndrome. Indian $J$ Pathol Microbiol, 52, 206-9.

Wang XQ. Sino-US Shanghai Leukemia Cooperative Group 
Sadia Sultan et al

(2008).WHO classification and cytogenetic analysis of 435 cases with myelodysplastic syndrome. Zhonghua Nei Ke Za Zhi, 47, 464-7.

Yan X, Wei J, Wang J, et al (2015). Analysis of the karyotype abnormalities and its prognostic in 298 patients with myelodysplastic syndrome. Zhonghua Хие Yе Хие Za Zhi, 36, 297-301. 